\title{
Aplicação do Whale Optimization Algorithm no Projeto de uma Estrutura Centralizada de Controle para o Amortecimento de Oscilações Eletromecânicas
}

\author{
Pedro M. Oliveira. Wesley Peres. \\ Francisco C. R. Coelho. \\ Programa de Pós-Graduação em Engenharia Elétrica \\ (PPGEL/UFSJ/CEFET-MG), Departamento de Engenharia Elétrica \\ (DEPEL), Universidade Federal de São João del-Rei (UFSJ), São \\ João-del Rei, MG, Brasil (e-mails: pe-droliveira@hotmail.com; \\ wesley.peres@ufsj.edu.br;franciscocoelho@ufsj.edu.br).
}

\begin{abstract}
This paper presents an application of the Whale Optimization Algorithm to design a hierarchical control structure for power system oscillation damping. The parameters of controllers are tuned in order to place the eigenvalues in closed loop operation into a suitable region of the complex plane. To ensure robustness, a set of operating conditions are taken into account. The control scheme is composed of two levels: the first one (local level) is based on the use of local signals and the second one (called centralized level) is based on the use of remote signals measured by phasor measurement units. Time delays are included in the design and a case study is conducted for a Brazilian Equivalent System.
\end{abstract}

Resumo: O presente trabalho apresenta a aplicação do Whale Optimization Algorithm no projeto de uma estrutura de controle hierárquica para o amortecimento de oscilações eletromecânicas em Sistemas Elétricos de Potência. Os parâmetros dos controladores são ajustados de forma a alocar os pólos de malha fechada em uma região adequada do plano complexo. Para a garantia de robustez, diversos cenários operativos são considerados no projeto. A estrutura de controle é composta por dois níveis: o nível local que utiliza sinais locais e o nível central que utiliza sinais remotamente medidos por Unidades de Medição Fasorial Sincronizada. Os tempos de atraso na transmissão de sinais são devidamente tratados na fase de projeto e um estudo de caso é conduzido para um sistema equivalente brasileiro.

Keywords: Hierarchical control; Power oscillation damping; Whale Optimization Algorithm; Grey Wolf Optimization; Power system stability.

Palavras-chaves: Controle hierárquico; Amortecimento de oscilações de potência; Whale Optimization Algorithm; Grey Wolf Optimization; Estabilidade de sistemas elétricos.

\section{INTRODUÇÃO}

Oscilações eletromecânicas de baixa frequência estão associadas à excursão do ângulo do rotor dos geradores na ocorrência de perturbações no sistema elétrico (pequenas ou grandes). De forma geral, tais oscilações são estudadas utilizando-se modelos linearizados do sistema elétrico, a partir da análise modal. A análise modal consiste na análise de autovalores (de suas frequências de oscilação e coeficientes de amortecimento). Com base na frequência de oscilação definem-se dois tipos principais de oscilações: $(i)$ oscilações locais entre um gerador e o resto do sistema (1 a 3 $\mathrm{Hz})$ e (ii) oscilações interarea entre dois grupos de geradores em áreas distintas do sistema $(0,2$ a 1,0 Hz) (Sauer \& Pai, 1998).

Tais oscilações causam desgastes mecânicos nos geradores e podem reduzir os limites de transferência de potência entre áreas. Nesse sentido, desde a década de setenta, Estabilizadores de Sistemas de Potência (ESP), controladores que atuam no sistema de excitação dos geradores, têm sido empregados (Demello \& Concordia, 1969). Tais controladores são compostos por estágios de ganho e de compensação de fase que devem ser ajustados levando-se em consideração as incertezas de carga e de topologia da rede elétrica.

Os ESPs são indicados para o amortecimento de oscilações locais. Para o amortecimento de oscilações interarea (um problema de natureza global), quando possível, comumente procede-se com o ajuste simultâneo dos diversos controladores do sistema (Dotta et al., 2009).

Outras alternativas para o amortecimento dos modos interarea são: (i) uso de sinais estabilizantes nos dispositivos FACTS e (ii) uso de sinais remotos na entrada dos ESPs dos geradores síncronos. O uso de sinais remotos é atrativo por estes possuírem maior observabilidade em relação aos modos interarea (Zhang \& Bose, 2008).

Uma das estruturas de controle baseadas em sinais remotos é a Hierárquica, composta por dois níveis. O primeiro nível é denominado de Controlador Local (também chamado de Descentralizado), que utiliza sinais locais de desvio de velocidade. O segundo, denominado Controlador Central, utiliza sinais remotos medidos por Unidades de Medição Fasorial Sincronizada (UMFS). Dessa forma, cada gerador que participe do Controle Hierárquico recebe dois sinais estabilizantes: um do controle local e outro do controle central (Dotta, 2009). As UMFS são equipamentos capazes 
de medir fasores de tensão e corrente em pontos geograficamente distantes em uma referência comum de tempo (Zhang \& Bose, 2008).

Conforme (Dotta et al., 2009; Cárdenas, 2011), a implementação do controle hierárquico não é justificada se o interesse for $\mathrm{o}$ aumento do amortecimento: o ajuste coordenado do controle descentralizado (local) é suficiente. Entretanto, se o ajuste coordenado não for possível devido a fatores técnicos e econômicos, o controle hierárquico é uma opção atrativa.

O problema de ajuste do controle hierárquico foi recentemente tratado utilizando técnicas de controle moderno (robusto) e técnicas de otimização paramétrica. Dentre os trabalhos utilizando controle moderno cita-se: Regulador Linear Quadrático (LQR) (Dotta et al., 2009) e Inequações Matriciais Lineares (LMI) (Zhang \& Bose, 2008).

Dentre os métodos de otimização paramétrica utilizados para o projeto do Controle Hierárquico cita-se: Método Híbrido (baseado em um acoplamento dos métodos Quasi-Newton, Feixes e Gradiente Amostrado) (Dotta, 2009), Algoritmos Genéticos $(A G)$ (Cárdenas, 2011) e Otimização baseada em Enxame de Partículas (PSO) (Peres, 2016).

$\mathrm{Na}$ linha dos trabalhos anteriormente mencionados, este artigo propõe a aplicação de uma metaheurística populacional, recentemente proposta na literatura, para a solução do problema de ajuste ótimo do controle hierárquico. A metaheurística é o Whale Optimization Algorithm (WOA), baseado no método de caça de Baleias Jubarte (Mirjalili \& Lewis, 2016). O objetivo é ajustar os parâmetros dos controladores considerando diversos cenários operativos com o objetivo de alocar os pólos em malha fechada em regiões adequadas do plano complexo. Os resultados são comparados com aqueles obtidos através de outra recente metaheurística proposta na literatura, denominada Grey Wolf Optimization, baseada no comportamento de lobos cinzentos na busca por alimento (Mirjalili et al., 2014).

\section{APRESENTAÇÃO DO PROBLEMA}

\subsection{Controle Hierárquico}

A Fig. 1 ilustra as estruturas de controle descentralizada e centralizada em um SEP composto de dois geradores que participam de ambos os níveis do controle hierárquico. No controle centralizado, baseado no uso de sinais remotos, o tempo de atraso na transmissão do sinal é considerado (Atraso). Observa-se que cada gerador recebe um sinal do controle local e um do controle central. O sinal de controle é utilizado para modular a tensão de referência do Regulador Automático de Tensão (RAT).

\subsection{Modelo do Estabilizador}

A função de transferência de cada estabilizador do controle hierárquico (em ambos os níveis) é apresentada na equação (1).

$$
\operatorname{ESP}_{p}(s)=K_{p} \cdot \frac{\left(s . T_{w}\right)}{\left(1+s \cdot T_{w}\right)} \cdot \frac{\left(1+s \frac{\sqrt{\alpha_{p}}}{\omega_{p}}\right)^{n b}}{\left(1+s \frac{1}{\omega_{p \cdot \sqrt{\alpha_{p}}}}\right)^{n b}}
$$

em que $T_{w}$ é a constante de tempo do filtro washout, usado para que o estabilizador atue somente no regime transitório (essa constante é conhecida) e $n b$ é o número de compensadores de atraso e/ou avanço utilizados (parâmetro também conhecido). Os parâmetros a serem ajustados para o controlador são: o ganho $K_{p}$, o parâmetro de compensação de fase $\alpha_{p}$ e a frequência onde ocorre a máxima compensação de fase $\omega_{p}$.

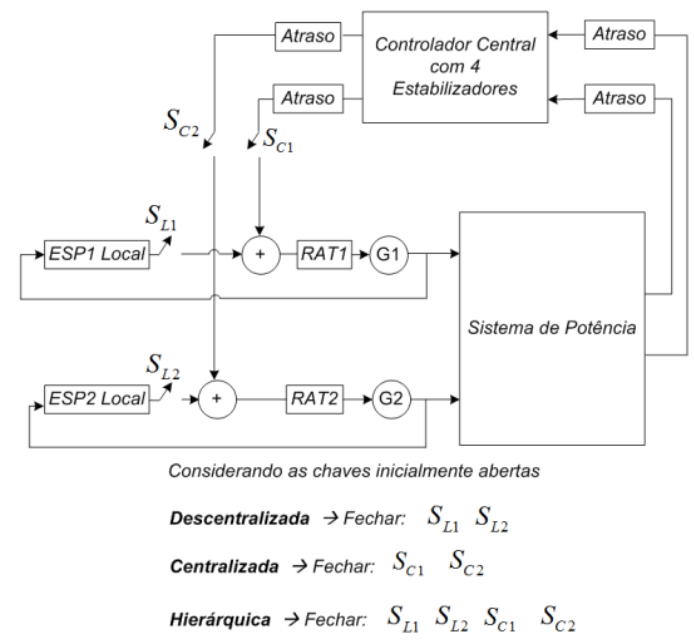

Fig. 1 Estruturas de controle consideradas. Fonte: (Dotta et al., 2009).

\subsection{Modelo do Sistema em Malha Aberta}

Para o projeto de controladores, o sistema de potência é modelado em espaço de estados (no domínio do tempo). Para isso, as equações que descrevem o comportamento dinâmico do sistema são linearizadas em torno de um ponto de operação $j$, conforme descrito na equação (2).

$$
\begin{aligned}
& \dot{x}=A_{a j} x+B_{a j} u \\
& y=C_{a j} x+D_{a j} u
\end{aligned}
$$

em que $x$ são as variáveis de estado (velocidade das máquinas, ângulos e tensões internas e de campo), $u$ representa as variáveis de entrada (tais como os sinais de controle: tensão de referência dos reguladores de tensão) e $y$ são as variáveis de saída (velocidade terminal das máquinas). As matrizes em malha aberta em uma condição operativa $j$ são: estados $\left(A_{a j}\right)$, entrada $\left(B_{a j}\right)$, saída $\left(C_{a j}\right)$ e transmissão direta $\left(D_{a j}\right)$.

Em malha aberta os geradores possuem somente os reguladores automáticos de tensão. Portanto o sistema não possui estabilizadores. A estrutura das matrizes da formulação em espaço de estados para o problema em questão pode ser obtida em (Sauer \& Pai, 1998).

A estabilidade do sistema em um determinado ponto de operação pode ser avaliada através do coeficiente de amortecimento dos autovalores da matriz de estados: os sistemas instáveis possuem coeficientes de amortecimento negativos (Sauer \& Pai, 1998). 


\subsection{Modelo das Estruturas de Controle}

Os controladores local e central podem ser expressos matricialmente com os seus respectivos estabilizadores. Considerando-se $q$ estabilizadores no controlador local (descentralizado), pode-se representar essa estrutura de controle por uma matriz diagonal $\operatorname{ESP}_{\text {local }}(s)$ conforme a equação (3).

$$
\operatorname{ESP}_{\text {local }}(s)=\left[\begin{array}{ccc}
E S P_{1}(s) & \ldots & 0 \\
\vdots & \ddots & \vdots \\
0 & \ldots & \operatorname{ESP}_{q}(s)
\end{array}\right]
$$

O controlador central pode ser representado por uma matriz genérica $\operatorname{ESP}_{\text {central }}(s)$ de ordem $(q \times p)$ conforme a equação (4). Nesse caso: $q$ é o número de saídas do controlador (número de geradores que recebem sinais do controlador central) e $p$ é o número de entradas do controlador (número de geradores que enviam seus sinais remotos de velocidade para o controle central).

$$
\operatorname{ESP}_{\text {central }}(s)=\left[\begin{array}{ccc}
E S P_{11}(s) & \ldots & E S P_{1 p}(s) \\
\vdots & \ddots & \vdots \\
E S P_{q 1}(s) & \ldots & E S P_{q p}(s)
\end{array}\right]
$$

\subsection{Modelo dos Tempos de Atraso}

O tempo de atraso na transmissão de sinais remotos foi modelado usando a Aproximação de Padé conforme a equação (5). Essa aproximação foi adotada em (Dotta et al., 2009; Cárdenas, 2011) e apresenta uma resposta nula em t = 0 , sendo adequada para a modelagem de sistemas com atraso.

$$
\mathrm{e}^{-\mathrm{sT}}=\frac{-(2 \mathrm{~T}) \cdot \mathrm{s}+6}{\left(\mathrm{~T}^{2}\right) \cdot \mathrm{s}^{2}+(4 \mathrm{~T}) \cdot \mathrm{s}+6}
$$

\subsection{Processo de Realimentação}

Neste trabalho, o processo de inclusão dos controladores no sistema (processo de realimentação) é feito em duas etapas. Inicialmente o controlador local é inserido no sistema. O controlador $\operatorname{ESP}_{\text {local }}(s)$ pode ser representado em espaço de estados conforme a equação (6).

$$
\begin{aligned}
& \dot{x_{c}}=A_{c} x_{c}+B_{c} u_{c} \\
& y_{c}=C_{c} x_{c}+D_{c} u_{c}
\end{aligned}
$$

em que $x_{c}$ são as variáveis de estado dos controladores, $u_{c}$ são os sinais de desvio de velocidade e $y_{c}$ são as variáveis com os sinais adicionais estabilizantes (tensões).

Em seguida, o controlador central é inserido considerando os tempos de atraso de transmissão do sinal. O controlador central $\operatorname{ESP}_{\text {central }}(s)$, considerando os tempos de atraso, também pode ser representado de acordo com a equação (6).

\subsection{Sistema em Malha Fechada}

O sistema em malha fechada para o ponto de operação $j$ considerando a estrutura de controle projetada será dada pela equação (7).

$$
\begin{gathered}
\dot{x_{f}}=A_{f j} x_{f}+B_{f j} u_{f} \\
y_{f}=C_{f j} x_{f}+D_{c} u_{f}
\end{gathered}
$$

em que o índice $f$ está associado à representação em malha fechada, que considera as variáveis de estado em malha aberta e dos controladores. Ressalta-se que o vetor de estados em malha fechada $x_{f}$ não possui a mesma dimensão do vetor de estados da planta em malha aberta $(x)$. A estrutura da matriz $A_{f j}$ é apresentada na equação (8).

$$
\left[A_{f j}\right]=\left[\begin{array}{cc}
A_{a j}+B_{a j} D_{c} C_{a j} & B_{a j} C_{c} \\
B_{c} C_{a j} & A_{c}
\end{array}\right]
$$

\subsection{Problema de Otimização}

Nesse trabalho, procede-se com a otimização do controle centralizado (segundo nível do controle hierárquico). Nesse caso, considera-se que o controle local já foi projetado baseando-se em regras das empresas de energia elétrica. O objetivo é ajustar os parâmetros de tal forma que os autovalores em malha fechada estejam alocados em regiões apropriadas no plano complexo. Para a garantia de robustez, são considerados vários cenários operativos na fase de projeto.

A função objetivo adotada nesse trabalho, dada pela equação (9), é composta por duas parcelas, apresentadas nas equações (10) e (11).

$$
\begin{gathered}
f o b=\beta \cdot J_{1}+(1-\beta) \cdot J_{2} \\
J_{1}=\sum_{j=1}^{n c e n a r i o s} \sum_{\sigma_{i, j} \geq \sigma_{0}}\left(\sigma_{0}-\sigma_{i, j}\right)^{2} \\
J_{2}=\sum_{j=1}^{n c e n a r i o s} \sum_{\xi_{i, j} \leq \xi_{0}}\left(\xi_{0}-\xi_{i, j}\right)^{2}
\end{gathered}
$$

em que: (i) ncenarios representa o número de cenários adotados na fase do projeto; ( $i i$ ) $\sigma_{0}$ e $\xi_{0}$ são a maior parte real e o mínimo amortecimento permitidos para os autovalores em malha fechada e (iii) $\sigma_{i, j}$ e $\xi_{i, j}$ são a parte real e o amortecimento do autovalor $i$ no cenário $j$ (considera-se no somatório somente os autovalores que não atendem aos requisitos de amortecimento mínimo e parte real máxima). Por fim, $\beta$ é um fator de peso. Como resultado do processo de otimização, espera-se que todos os autovalores complexos (ou de natureza eletromecânica) sejam alocados no interior de uma região adequada do plano complexo, ilustrada na Fig. 2.

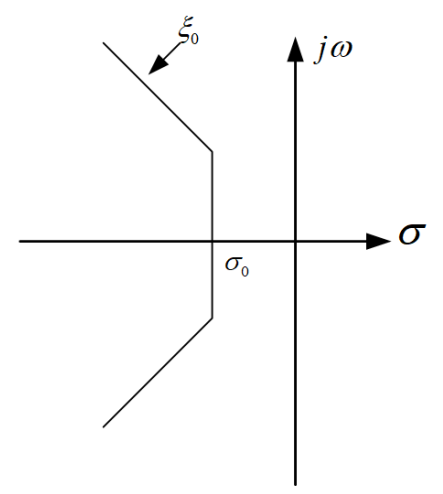

Fig. 2 Região definida no plano complexo em que $\xi>\xi_{0}$ e

$$
\boldsymbol{\sigma}<\boldsymbol{\sigma}_{\mathbf{0}}
$$

A formulação do problema de otimização é apresentada na equação (12). 


$$
\left\{\begin{array}{cc}
\text { Min } & f o b \\
& K_{p \min } \leq K_{p} \leq K_{p \max } \\
\text { s.a. } & \alpha_{p \min } \leq \alpha_{p} \leq \alpha_{p \max } \\
& \omega_{p \min } \leq \omega_{p} \leq \omega_{p \max }
\end{array}\right.
$$

em que as restrições de canalização representam o espaço de busca considerando os limites dos parâmetros a serem ajustados para cada estabilizador.

\subsection{Estrutura dos Indivíduos e Cálculo da Função Objetivo}

Para a solução do problema de otimização utiliza-se duas metaheurísticas populacionais: (i) Whale Optimization Algorithm (Mirjalili \& Lewis, 2016) e (ii) Grey Wolf Optimization (Mirjalili et al., 2014). A estrutura de cada indivíduo da população é representada na equação (13).

$$
x_{k}=\left[\begin{array}{lllllll}
\mathrm{K}_{1} & \ldots & \mathrm{K}_{\mathrm{p}} \mid \alpha_{1} & \ldots & \alpha_{\mathrm{p}} \mid \omega_{1} & \ldots & \omega_{\mathrm{p}}
\end{array}\right]
$$

Para cada indivíduo representado em (13): para um determinado ponto de operação $j$, procede-se com o processo de realimentação descrito na seção 2.6 e, ao final, a matriz em espaço de estados em malha fechada será conforme (7). O processo é repetido para os demais pontos de operação e ao final, calculam-se todos os autovalores em todos os pontos de operação. De posse dos autovalores, calcula-se a função objetivo ou aptidão dada em (9).

\section{WHALE OPTIMIZATION ALGORITHM (WOA)}

O Whale Optimization Algorithm (WOA) é baseado no método de caça de Baleias Jubarte (Mirjalili \& Lewis, 2016). Esse procedimento (ilustrado na Fig. 3), conhecido como método de alimentação por rede de bolhas, é usado para a captura de pequenos peixes e krills (pequenos crustáceos) que ficam próximos à superfície.

As redes de bolhas funcionam como uma espécie de barreira, impedindo o escape do cardume e ajudando na captura. Em seguida, a baleia enche a boca com o cardume e água devolvendo a água em excesso ao mar usando suas barbatanas. Assim, a baleia engole somente o cardume, filtrando o alimento (Mirjalili \& Lewis, 2016).

Adicionalmente cita-se o comportamento social das baleias, que caçam em grupos. Nesse sentido pode-se modelar um algoritmo de otimização populacional baseado na inteligência coletiva e troca de informações entre indivíduos.

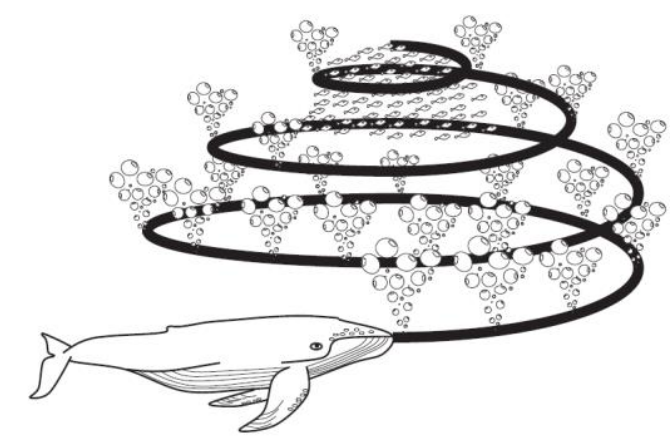

Fig. 3 Método de alimentação por rede de bolhas. Fonte: (Mirjalili \& Lewis, 2016).

\subsection{Modelagem Matemática do Procedimento de Cercar a Presa}

As baleias reconhecem a localização da presa e a cercam. Fazendo analogia a um problema de otimização, o procedimento de caça seria a busca pela melhor solução (presa). Em um problema de otimização não se conhece, a priori, a melhor solução (posição da presa). Assim, o WOA assume que a melhor solução corrente $\vec{X}_{p}(t)$ é a posição da presa (ou que $\vec{X}_{p}(t)$ é uma boa indicação da posição da presa).

Uma vez identificada a melhor solução corrente $\vec{X}_{p}(t)$ os demais indivíduos (baleias) se movimentam em direção à melhor solução, atualizando suas posições conforme as equações (14) a (17):

$$
\begin{gathered}
\vec{D}=\left|\vec{C} \cdot \vec{X}_{p}(t)-\vec{X}(t)\right| \\
\vec{X}(t+1)=\vec{X}_{p}(t)-\vec{A} \cdot \vec{D} \\
\vec{A}=2 \vec{a} \cdot \vec{r}_{1}-\vec{a} \\
\vec{C}=2 \cdot \vec{r}_{2}
\end{gathered}
$$

em que $t$ é a iteração atual, $t_{\max }$ é o número máximo de iterações, $\vec{A}$ e $\vec{C}$ são coeficientes vetoriais, $\vec{X}_{p}$ é o vetor de posição da presa (melhor solução corrente), $\vec{X}$ é o vetor de posição da baleia, $\vec{r}_{1}$ e $\vec{r}_{2}$ são vetores randômicos no intervalo de $[0,1]$, | | indica valor absoluto e $\vec{a}$ é um vetor linearmente diminuído de 2 à 0 no decorrer das iterações. A dimensão de cada indivíduo (baleia) foi ilustrada na equação (13) para o caso de $p$ estabilizadores.

\subsection{Busca Local: Ataque por Redes de Bolha}

Duas abordagens são possíveis no ataque por redes de bolha:

\section{i. Mecanismo de Circundamento Decrescente} (Shrinking encircling mechanism) - esse comportamento é obtido a partir da redução do valor do $\vec{a}$ na equação (16). Nota-se que a variação de $\vec{A}$ também é reduzida com $\vec{a}$. Em outras palavras, $\vec{A}$ é um vetor de valores aleatórios dentro do intervalo de $[-a, a]$ onde $a$ é um valor entre 2 e 0 que é decrescido linearmente ao decorrer das iterações conforme a equação (18). Ao definir valores aleatórios para $\vec{A}$ entre $[-1,1]$, a nova posição para o indivíduo pode encontrar-se em qualquer lugar entre sua posição original e a posição do melhor indivíduo.

$$
a=2-2 \cdot t / t_{\max }
$$

ii. Atualização da Posição em Espiral (Spiral updating position) - uma vez definida a distância entre a posição de uma baleia $\vec{X}(t)$ e a melhor solução corrente $\vec{X}_{p}(t)$, um movimento helicoidal é simulado através das equações (19) a (20).

$$
\begin{gathered}
\overrightarrow{D^{\prime}}=\left|\vec{X}_{p}(t)-\vec{X}(t)\right| \\
\vec{X}(t+1)=\overrightarrow{D^{\prime}} \cdot e^{b l} \cdot \cos (2 \pi l)+\vec{X}_{p}(t)
\end{gathered}
$$


em que $b$ é uma constante que define o formato espiral logaritmo e $l$ é um número randômico no intervalo $[-1,1]$.

Considerando que existem duas possibilidades de movimento na busca local (ataque à presa), é considerado que o indivíduo vai se movimentar de uma forma ou outra conforme uma probabilidade. A equação (21) modela esse procedimento, onde $\mathrm{p}$ é um número randômico de distribuição uniforme no intervalo $[0,1]$.

$$
\vec{X}(t+1)= \begin{cases}\vec{X}_{p}(t)-\vec{A} \cdot \vec{D} & \text { se } p<0,5 \\ \overrightarrow{D^{\prime}} \cdot e^{b l} \cdot \cos (2 \pi l)+\vec{X}_{p}(t) & \text { se } p \geq 0,5\end{cases}
$$

\subsection{Busca Global: Exploração do Espaço de Busca}

A identificação de regiões mais promissoras é chamada de busca global. No WOA corresponde isso à capacidade de explorar o espaço de busca com o objetivo de encontrar novas fontes de alimento.

Nessa etapa, usa-se o vetor $\vec{A}$ com valores aleatórios maiores que 1 ou menores que -1 para forçar o indivíduo a se mover para longe da baleia de referência $\left(\vec{X}_{p}(t)\right)$. Em contraste com a fase de busca local, atualiza-se a posição do indivíduo na fase de busca global de acordo com uma baleia aleatoriamente escolhida, ao invés do indivíduo que obteve o melhor resultado até então $\overrightarrow{\mathrm{X}}_{\mathrm{p}}(\mathrm{t})$. As equações (22) a (23) modelam essa etapa de busca global:

$$
\begin{gathered}
\vec{D}=\left|\vec{C} \cdot \vec{X}_{\text {rand }}(t)-\vec{X}(t)\right| \\
\vec{X}(t+1)=\vec{X}_{\text {rand }}(t)-\vec{A} \cdot \vec{D}
\end{gathered}
$$

em que $|\vec{A}|>1$ e $\vec{X}_{\text {rand }}(t)$ é a posição de um indivíduo escolhido randomicamente na população.

\subsection{Algoritmo}

O algoritmo básico do WOA é apresentado na Fig. 4.

\section{ESTUDO DE CASO}

Nessa seção é apresentado um estudo de caso para um sistema equivalente brasileiro. Foi utilizada a plataforma MATLAB $^{\circledR}$ e um computador com as seguintes configurações: processador Intel ${ }^{\circledR}$ Core i7-8550U, $1,80 \mathrm{GHz}$ com 12 GB de memória RAM e sistema operacional Windows 10-64 bits.

\subsection{Descrição do Sistema em Malha Aberta}

O diagrama unifilar do sistema equivalente Sul-Sudeste é apresentado na Fig. 5.

Este sistema é composto por cinco máquinas síncronas, sendo que a máquina da barra 7 representa o sistema equivalente da região sudeste do Brasil (portanto não possui estabilizador). Os reguladores de tensão são do tipo estático (com baixa constante de tempo e alto ganho). Os dados completos desse sistema podem ser obtidos em (Martins, 1986). A análise modal desse sistema indica dois modos interarea críticos: um associado à oscilação entre Sudeste e Itaipu e outro associado

\begin{tabular}{|c|c|}
\hline Algoritmo WOA & $\underline{\text { Passo }}$ \\
\hline Início & 1 \\
\hline $\begin{array}{l}\text { Inicializar a população de baleias } X_{i}(i= \\
1,2, \ldots, n) \text { dentro dos limites inferior e } \\
\text { superior das variáveis }\end{array}$ & 2 \\
\hline Calcular a aptidão de cada indivíduo & 3 \\
\hline$\vec{X}_{p}=$ o melhor indivíduo & 4 \\
\hline Inicializar o contador de iterações $t \leftarrow 1$ & 5 \\
\hline $\begin{array}{l}\text { Enquanto }(t<\text { número máximo de iterações) } \\
\text { Faça }\end{array}$ & 6 \\
\hline Para cada indivíduo Faça & 7 \\
\hline Atualizar $a, A, l$ e $p$ & 8 \\
\hline Se1 $(p<0,5)$ Faça & 9 \\
\hline $\operatorname{Se} 2(|\mathrm{~A}|<1)$ Faça & 10 \\
\hline $\begin{array}{l}\text { Atualizar a posição do } \\
\text { indivíduo usando a equação } \\
(15)\end{array}$ & 11 \\
\hline Senão Se2 (|A| $\geq 1)$ Faça & 12 \\
\hline $\begin{array}{l}\text { Selecionar aleatoriamente um } \\
\text { indivíduo da população } \vec{X}_{\text {rand }}\end{array}$ & 13 \\
\hline $\begin{array}{l}\text { Atualizar a posição do } \\
\text { indivíduo usando a equação } \\
(23)\end{array}$ & 14 \\
\hline Fim Se2 & 15 \\
\hline Senão Se1 $(p \geq 0,5)$ Faça & 16 \\
\hline $\begin{array}{l}\text { Atualizar a posição do indivíduo } \\
\text { usando a equação (20) }\end{array}$ & 17 \\
\hline Fim Se1 & 18 \\
\hline $\begin{array}{l}\text { Caso alguma variável tenha } \\
\text { extrapolado o limite inferior ou } \\
\text { superior, colocar a respectiva variável } \\
\text { no limite violado. }\end{array}$ & 19 \\
\hline Calcular a aptidão do indivíduo & 20 \\
\hline $\begin{array}{l}\text { Atualizar } \vec{X}_{p} \text { se uma melhor solução } \\
\text { for encontrada. }\end{array}$ & 21 \\
\hline Fim-Para & 22 \\
\hline$t \leftarrow 1+1$ & 23 \\
\hline Fim Enquanto & 24 \\
\hline Retornar $\vec{X}_{p}$ & 25 \\
\hline Fim & 26 \\
\hline
\end{tabular}

à oscilação entre o sistema Sul (G1, G2 e G3) e Sudeste mais Itaipu.

Fig. 4 Algoritmo básico do WOA.

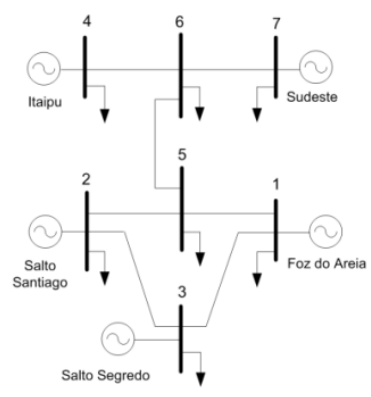

Fig. 5 Sistema equivalente Sul-Sudeste. 
O procedimento de ajuste é realizado considerando cinco pontos de operação pré-selecionados para o sistema, descritos na Tabela 1. O mínimo amortecimento em malha aberta para cada ponto é apresentado: observa-se que o sistema é instável.

Tabela 1. Característica em Malha Aberta.

\begin{tabular}{|c|cc|c|}
\hline Caso & \multicolumn{2}{|c|}{ Configuração } & $\xi_{\min }(\%)$ \\
\hline 1 & $\mathrm{X}_{5-6}=0,39 \mathrm{pu}$ & $\mathrm{X}_{6-7}=0,57 \mathrm{pu}$ & $-11,90$ \\
\hline 2 & $\mathrm{X}_{5-6}=0,50 \mathrm{pu}$ & $\mathrm{X}_{6-7}=0,57 \mathrm{pu}$ & $-12,10$ \\
\hline 3 & $\mathrm{X}_{5-6}=0,80 \mathrm{pu}$ & $\mathrm{X}_{6-7}=0,57 \mathrm{pu}$ & $-12,66$ \\
\hline 4 & $\mathrm{X}_{5-6}=0,39 \mathrm{pu}$ & $\mathrm{X}_{6-7}=0,63 \mathrm{pu}$ & $-14,04$ \\
\hline 5 & $\mathrm{X}_{5-6}=0,39 \mathrm{pu}$ & $\mathrm{X}_{6-7}=0,70 \mathrm{pu}$ & $-16,59$ \\
\hline
\end{tabular}

\subsection{Inclusão do Controle Local}

O controlador local (descentralizado) pode ser ajustado utilizando-se a metodologia descrita na seção 2. Entretanto, o mesmo procedimento adotado em (Dotta et al., 2009) será utilizado: parte-se do princípio que as empresas configuraram os seus respectivos estabilizadores de acordo com métodos de projeto normatizados pela concessionária. Nesse sentido, consideram-se os parâmetros apresentados na Tabela 2.

Tabela 2. Controle Local.

\begin{tabular}{|c|c|c|c|}
\hline Geradores & $\boldsymbol{K}_{\boldsymbol{p}}$ & $\boldsymbol{\alpha}$ & $\boldsymbol{\omega}$ \\
\hline 03 & 14,862 & 5,006 & 6,404 \\
\hline 04 & 14,442 & 8,113 & 4,970 \\
\hline \multicolumn{2}{|l|}{$\begin{array}{l}\text { São considerados dois blocos de compensação de fase e a } \\
\text { constante de tempo do filtro washout é igual a } 3 \text { segundos }\end{array}$} \\
\hline
\end{tabular}

Com o controle local, o sistema é estável nas cinco condições operativas consideradas. Entretanto um amortecimento mínimo de $4,5 \%$ é obtido. No presente trabalho, a melhoria do amortecimento será feita através do ajuste do controlador central utilizando os métodos WOA e GWO.

\subsection{Estrutura do Controle Central}

A Fig. 6 apresenta o esquema do controlador centralizado, composto por 4 estabilizadores (dois geradores participam do controle central, G3 e G4). Existem, portanto, 12 parâmetros a serem otimizados. Inicialmente os sinais remotos de velocidade são medidos pelas Unidades de Medição Fasorial Sincronizada (UMFS) e enviados ao controlador central. Em seguida, os sinais processados no controle central são reenviados para os geradores.
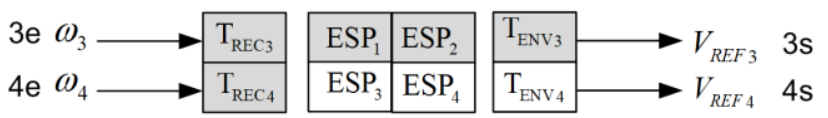

Fig. 6 Estrutura do controlador central.

Os tempos de recepção dos sinais remotos $\left(\mathrm{T}_{\mathrm{REC}}=200 \mathrm{~ms}\right) \mathrm{e}$ de envio do sinal de controle $\left(\mathrm{T}_{\mathrm{ENV}}=200 \mathrm{~ms}\right)$ são considerados. Como exemplo, o sinal de controle a ser injetado no gerador 3 é uma composição dos sinais remotos processados no controlador central, conforme destacado na Fig. 6. Os índices (3s) e (3e) indicam, respectivamente, a primeira saída do controlador central (a ser injetada em G3) e a primeira entrada (sinal remoto medido em G3). A mesma notação é aplicada aos demais sinais.
Para efeitos de informação, a dimensão da matriz de estados é igual: a 30 em malha aberta, 36 em malha fechada com o controle local e 56 em malha fechada com o controle hierárquico.

As seguintes constantes são associadas ao controlador central: $T_{w}=3$ seg e $n b=2$. Os limites das variáveis são dados pela equação (24).

$$
\begin{aligned}
& 0,01 \leq K_{p} \leq 20,00 \\
& 0,10 \leq \alpha_{p} \leq 10,00 \\
& 1,25 \leq \omega_{p} \leq 30,00
\end{aligned}
$$

São considerados 25 indivíduos e 50 gerações. Os valores necessários para o cálculo da função objetivo são: $\sigma_{0}=-1 \mathrm{e}$ $\xi_{0}=0,1$. Isso significa, por exemplo, que o amortecimento mínimo (considerando todos os cenários operativos) deve ser maior que $10 \%$.

\subsection{Resultados}

A Tabela 3 apresenta os resultados obtidos pelo GWO e $W O A$ para diversos valores de $\beta: f o b=\beta . J_{1}+(1-\beta) . J_{2}$, em que $J_{2}$ está associado ao coeficiente de amortecimento. Em relação ao mínimo coeficiente de amortecimento obtido pelo GWO (considerando todos os cenários operativos), o valor deste parâmetro reduz com o aumento de $\beta$, o que era esperado: em contrapartida, observa-se o aumento da parte real do autovalor dominante (em módulo). É visível, pois, que o ajuste no valor de $\beta$ permite enfatizar a característica de desempenho desejada.

Observa-se também uma melhora do $\xi_{\text {min }}$ quando se usa o WOA. Adicionalmente, nota-se que não foi possível atender os pré-requisitos de amortecimento mínimo (maior que 10\%) e parte real máxima maior que -1,00. Entretanto ressalta-se que os valores de $\xi_{\text {min }}$ são próximos ao obtido em (Dotta et al., 2009): um valor de 9,67\% para os mesmos cenários operativos foi obtido utilizando controle ótimo $L Q R$, que utilizou 16 estabilizadores no controle central, isto é, quando todos os geradores participam do segundo nível de controle. Ou seja, foi possível obter um resultado quase tão eficaz com um número menor de estabilizadores e geradores envolvidos.

Tabela 3. Resultados em função do $\boldsymbol{\beta}$.

\begin{tabular}{|c|c|c|c|}
\hline$\beta$ & & $G W O$ & $W O A$ \\
\hline \multirow{2}{*}{0} & $\xi_{\min }(\%)$ & 9,4233 & 8,3984 \\
\cline { 2 - 4 } & $\sigma_{\max }$ & $-0,5094$ & $-0,4425$ \\
\hline \multirow{2}{*}{0,25} & $\xi_{\min }(\%)$ & 7,9771 & 8,1762 \\
\cline { 2 - 4 } & $\sigma_{\max }$ & $-0,9663$ & $-0,9934$ \\
\hline \multirow{2}{*}{0,50} & $\xi_{\min }(\%)$ & 7,7435 & 8,0909 \\
\cline { 2 - 4 } & $\sigma_{\max }$ & $-1,4032$ & $-0,9840$ \\
\hline \multirow{2}{*}{0,75} & $\xi_{\min }(\%)$ & 7,2448 & 8,2326 \\
\cline { 2 - 4 } & $\sigma_{\max }$ & $-1,3104$ & $-1,0001$ \\
\hline \multirow{2}{*}{1,00} & $\xi_{\min }(\%)$ & 6,8821 & 8,2327 \\
\cline { 2 - 4 } & $\sigma_{\max }$ & $-1,2410$ & $-1,0001$ \\
\hline
\end{tabular}

A Fig. 7 apresenta o desvio de velocidade de G3 (Salto Segredo) em relação ao G4 (Itaipu) considerando um degrau de $1 \%(0,01 \mathrm{pu})$ na tensão de referência do regulador de tensão de G3 (em 1 seg). Foi considerada a solução obtida para $\beta=0,25$ no primeiro cenário. Observa-se uma similaridade das respostas obtidas para ambos os métodos, 
confirmando os parâmetros da Tabela 3. Os parâmetros dos controladores (central) são apresentados na Tabela 4.

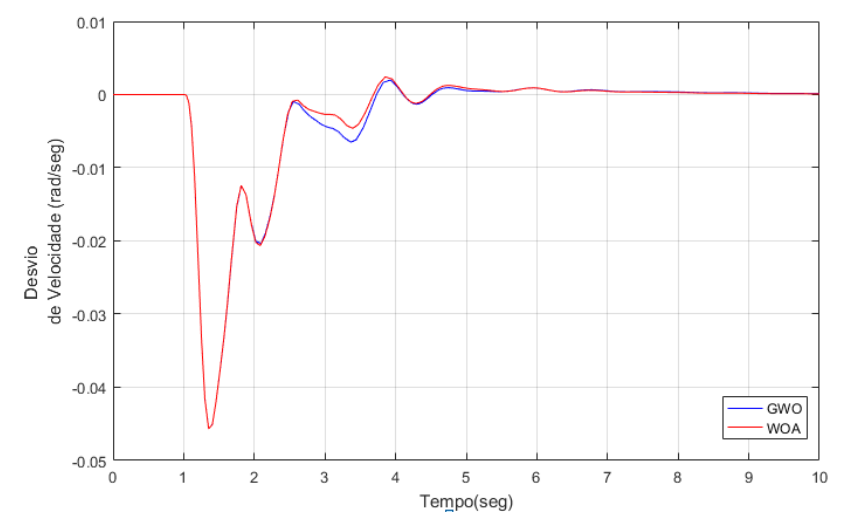

Fig. 7 Resposta temporal.

Tabela 4. Parâmetros de ajuste.

\begin{tabular}{|c|c|c|c|c|c|c|c|}
\hline \multicolumn{4}{|c|}{$\boldsymbol{W} \boldsymbol{O}$} & \multicolumn{4}{|c|}{ WOA } \\
\hline ESP & $\boldsymbol{K}_{\boldsymbol{p}}$ & $\boldsymbol{\alpha}$ & $\boldsymbol{\omega}$ & $\mathbf{E S P}$ & $\boldsymbol{K}_{\boldsymbol{p}}$ & $\boldsymbol{\alpha}$ & $\boldsymbol{\omega}$ \\
\hline 01 & 20,00 & 9,87 & 11,95 & 01 & 20,00 & 10,00 & 12,05 \\
\hline 02 & 7,56 & 0,21 & 8,20 & 02 & 8,00 & 0,10 & 20,66 \\
\hline 03 & 0,34 & 6,05 & 16,66 & 03 & 5,86 & 0,10 & 7,44 \\
\hline 04 & 20,00 & 0,12 & 28,59 & 04 & 8,81 & 0,38 & 17,17 \\
\hline
\end{tabular}

\section{CONCLUSÃO}

O presente trabalho apresentou a aplicação do Whale Optimization Algorithm (WOA) no ajuste dos parâmetros do controle centralizado, segundo nível do controle hierárquico para o amortecimento de oscilações eletromecânicas em sistemas elétricos. Diferentes cenários operativos foram considerados na fase do projeto, bem como os tempos de atraso na transmissão do sinal foram devidamente modelados. Os parâmetros foram ajustados com o objetivo de alocar os autovalores em malha fechada em uma região adequada do plano complexo definida pelo mínimo coeficiente de amortecimento e a máxima parte real de autovalores.

Os resultados foram comparados com aqueles obtidos pelo Grey Wolf Optimization $(G W O)$ e os resultados obtidos pelo WOA são competitivos.

Como proposta de continuidade é sugerido o desenvolvimento de metodologias híbridas de otimização para a melhoria dos resultados desses métodos. Com isso, pode-se considerar sistemas de controle de maior porte.

\section{AGRADECIMENTOS}

Os autores agradecem à FAPEMIG (projeto APQ-02245-18), CAPES (Finance Code 001), CNPq e PPGEL/UFSJ pelo auxílio financeiro. Agradecimentos também são prestados ao CEPEL pela autorização no uso das versões acadêmicas do programa PacDyn.

\section{REFERÊNCIAS}

Cárdenas, S.L.E., 2011. Projeto de Estabilizadores de Sistemas de Potência utilizando Sinais Remotos via Algoritmos Genéticos. Ph.D. dissertation.

Demello, F.P. \& Concordia, C., 1969. Concepts of Synchronous Machine Stability as Affected by Excitation Control. IEEE Transactions on Power Apparatus and Systems, PAS-88, pp.316-29.

Dotta, D., 2009. Controle Hierárquico usando Sinais de Medição Fasorial Sincronizada (Doutorado em Engenharia Elétrica). Ph.D. dissertation.

Dotta, D., e Silva, A.S. \& Decker, I.C., 2009. Wide-Area Measurements-Based Two-Level Control Design Considering Signal Transmission Delay. Power Systems, IEEE Transactions on, 24, pp.208-16.

Martins, N., 1986. Efficient Eigenvalue and Frequency Response Methods Applied to Power System SmallSignal Stability Studies. Power Systems, IEEE Transactions on, 1, pp.217-24.

Mirjalili, S. \& Lewis, A., 2016. The Whale Optimization Algorithm. Advances in Engineering Software, 95, pp.51-67..

Mirjalili, S., Mirjalili, S.M. \& Lewis, A., 2014. Grey Wolf Optimizer. Advances in Engineering Software, 69, pp.46-61..

Peres, W., Silva.V.V.R., Silva Junior I.C.and Passos.Filho.J.A., 2016. Projeto de Estabilizadores de Sistemas de Potência utilizando Sinais Remotos via PSO. In XXI Congresso Brasileiro de Automática, 2016, Vitória, ES., 2016.

Sauer, P. \& Pai, M.A., 1998. Power System Dynamics and Stability. Prentice Hall.

Zhang, Y. \& Bose, A., 2008. Design of Wide-Area Damping Controllers for Interarea Oscillations. IEEE Transactions on Power Systems, 23, pp.1136-43. 